\title{
Ladder-Type Silsesquioxane Copolymer Gate Dielectrics for High-Performance Organic Transistors
} and Inverters

Woonggi Kang, ${ }^{1}$ Gukil An, ${ }^{2}$ Min Je Kim, ${ }^{1}$ Wi Hyoung Lee, ${ }^{3}$ Dong Yun Lee, ${ }^{4}$ Hyunjung Kim, ${ }^{2}$ Jeong Ho $\mathrm{Cho}^{1 *}$

${ }^{1}$ SKKU Advanced Institute of Nanotechnology (SAINT), School of Chemical Engineering, Sungkyunkwan University, Suwon 440-746, Republic of Korea

${ }^{2}$ Department of Physics, Sogang University, Seoul 121-742, Republic of Korea

${ }^{3}$ Department of Organic and Nano System Engineering, Konkuk University, Seoul 143-701, Korea

${ }^{4}$ Department of Polymer Science and Engineering, Kyungpook National University, Daegu, 41566, Korea

*Corresponding author: jhcho94@,skku.edu

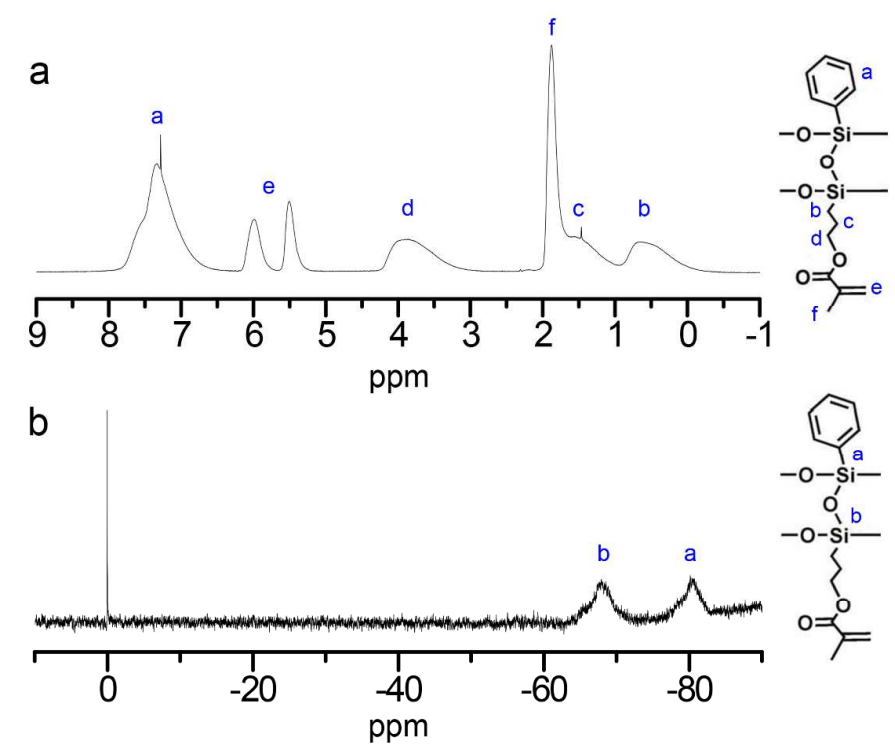

Figure S1. (a) ${ }^{1} \mathrm{H}$ NMR and (b) Si NMR spectra of the PPMSQ.

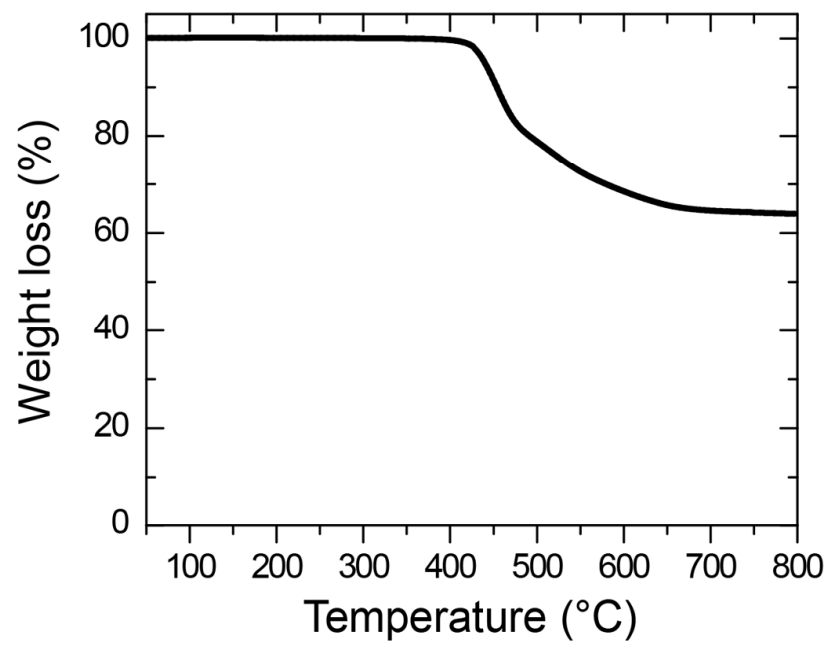

Figure S2. TGA thermogram of the PPMSQ. 